\title{
Primary peritonitis due to group A Streptococcus in a previously healthy pediatric patient
}

\author{
R Holden $M D^{1}$, A Wilmer $\mathrm{MD}^{2}$, $\mathrm{T}$ Kollman $M \mathrm{MD} \mathrm{PhD}^{3}$
}

\begin{abstract}
R Holden, A Wilmer, T Kollman. Primary peritonitis due to group A Streptococcus in a previously healthy pediatric patient. Can J Infect Dis Med Microbiol 2012;23(3):e69-70.
\end{abstract}

Primary peritonitis remains a rare disease in otherwise healthy children, with group A Streptococcus (GAS) being a particularly unusual cause. A case involving a 14-year-old girl, who presented with an 'acute abdomen' and was taken to the operating room for urgent laparoscopy, is reported. Abdominal and pelvic structures were only minimally inflamed, as was the appendix. Peritoneal fluid and blood cultures both grew pure cultures of GAS. The patient's course was complicated by streptococcal toxic shock syndrome. She fortunately made a full recovery. The present report highlights the diagnostic and treatment dilemmas associated with GAS primary peritonitis.

Key Words: Pediatrics; Peritonitis; Streptococcus pyogenes

\author{
Une péritonite primaire causée par un streptocoque \\ du groupe $\mathrm{A}$ chez un patient pédiatrique \\ auparavant en santé
}

La péritonite primaire demeure une maladie rare chez un enfant autrement en santé, et le streptocoque du groupe A (SGA) en est une cause particulièrement inhabituelle. Les auteurs présentent le cas d'une fille de 14 ans qui a consulté en raison d'un « abdomen aigu » et a dû subir une laparoscopie d'urgence en salle d'opération. Les structures abdominale et pelvienne, de même que l'appendice, étaient peu enflammées. Le liquide péritonéal et les prélèvements sanguins ont tous deux permis d'obtenir des cultures pures de SGA. L'évolution de la patiente a été compliquée par un syndrome de choc toxique streptococcique. Heureusement, elle s'est entièrement rétablie. Le présent rapport souligne les dilemmes diagnostiques et thérapeutiques associés à la péritonite primaire à SGA.

\section{CASE PRESENTATION}

A 14-year-old Asian girl was brought to the emergency department

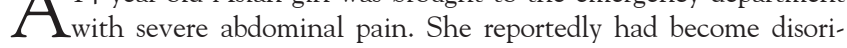
ented after a four-day history that began with one day of abdominal pain and was followed by three days of vomiting, nonbloody diarrhea and fever. The review of systems was otherwise negative, and she denied any travel history or animal exposures. She was previously healthy, with only a history of mild migraines, and she was not taking any regular medications. Her most recent menstrual period was one week before, and she denied any sexual activity.

When she presented at 06:00, her vitals included a heart rate of 150 beats/min, blood pressure of $91 / 61 \mathrm{mmHg}$, respiratory rate of 16 breaths $/ \mathrm{min}$ and a temperature of $38.5^{\circ} \mathrm{C}$. On examination in the emergency room, she was found to have a distended and rigid abdomen, which was most tender in the lower quadrants. Her examination was normal aside from cool extremities. Her white blood cell count on admission was normal $\left(10.8 \times 10^{9} / \mathrm{L}\right)$. Her C-reactive protein level was $274 \mathrm{mg} / \mathrm{L}$ and her electrolytes were consistent with acute renal failure, with sodium, potassium, urea and creatinine levels of $118 \mathrm{mmol} / \mathrm{L}$, $3.1 \mathrm{mmol} / \mathrm{L}, 21 \mathrm{mmol} / \mathrm{L}$ and $248 \mu \mathrm{mol} / \mathrm{L}$, respectively. Urinalysis and $\beta$-human chorionic gonadotropin were both negative. Her initial blood cultures were taken at $06: 30$ before the initiation of antibiotics.

The patient received $3 \mathrm{~L}$ of crystalloid fluid resuscitation, as well as doses of metronidazole at 07:20 and ciprofloxacin at 08:10 for presumed sepsis. An abdominal ultrasound failed to visualize her appendix, but did demonstrate free fluid. After being seen by the surgical team, imipenem was added at 11:30 and subsequently switched to meropenem after the first dose. She was taken to the operating room emergently, with a presumptive diagnosis of ruptured acute appendicitis.

Laparoscopic examination demonstrated frank pus throughout the abdomen and pelvis. Samples of peritoneal fluid were sent for culture at 16:30, approximately $9 \mathrm{~h}$ after the initiation of antibiotic therapy. The appendix was identified and described as only mildly inflamed, but not perforated. No other obvious source of infection could be identified after careful examination of the entire abdomen and pelvis. An intraoperative gynecological consult was also obtained and found no obvious pathology, aside from slightly erythematous and edematous pelvic structures, in keeping with diffuse peritonitis. The patient's abdomen was closed, with three drains left in situ, and clindamycin was added to the antimicrobial coverage at 08:00 on day 2 .

\begin{abstract}
Diagnosis
Peritoneal fluid collected during surgery revealed 4+ Gram-positive cocci suggestive of Streptococcus species. After overnight incubation, peritoneal fluid cultures grew beta hemolytic group A Streptococcus (GAS), confirming a diagnosis of GAS primary peritonitis. The blood cultures taken $10 \mathrm{~h}$ before surgery also grew GAS. GAS was not isolated from any other body site, including a vaginal culture at 18:00 on day 1 or an endotrachial aspirate culture on day 4 , although these were taken after initiation of imipenem. Despite the addition of ampicillin at 20:00 on day 2, the patient became progressively tachycardic and hypoxic to such an extent that her care was transferred to a pediatric intensive care unit approximately $48 \mathrm{~h}$ after initial receipt of ciprofloxacin and metronidazole in the emergency room. At that point, at 12:30 on day 3, her antibiotics were switched from meropenem and clindamycin to penicillin and clindamycin. Her chest $\mathrm{x}$-ray showed opacification of the lung fields bilaterally and she received bilevel positive airway pressure and was subsequently intubated for management of acute respiratory distress syndrome. Her presentation at this point was consistent with a 'definite' clinical diagnosis of GAS toxic shock syndrome (TSS) based on isolation of GAS from a normally sterile site (peritoneum), hypotension (systolic blood pressure of $70 \mathrm{mmHg}$ ), platelets of $31 \times 10^{9} / \mathrm{L}$ and acute respiratory distress syndrome. Her condition continued to deteriorate with her systolic blood pressure dropping to $70 \mathrm{mmHg}$, which required pressure support with epinephrine and norepinephrine. On day 4 , she was found to have an elevated troponin level and an echocardiogram showed globally impaired myocardial function with a left ventricular ejection fraction of less than $20 \%$ and a small pericardial effusion. One dose of intravenous immunoglobulin (IVIG) $(1 \mathrm{~g} / \mathrm{kg})$ was administered on day 4 , approximately $24 \mathrm{~h}$ after admission to the pediatric intensive care unit. The patient showed gradual hemodynamic improvement and ultimately recovered over a course of approximately 10 days, with her cardiac function improving (left ventricular ejection fraction 53\%) by day 8 . Intravenous penicillin was continued for 11 days, then the patient was stepped down to oral amoxicillin for a seven-day course.
\end{abstract}




\section{DISCUSSION}

Peritonitis involves inflammation of the peritoneum, and is typically described as primary, secondary or catheter related. Secondary peritonitis, due to perforation or leakage from an abdominal viscus, and catheterrelated peritonitis, related to indwelling catheters such as peritoneal dialysis catheters, occur more frequently in the pediatric population; primary peritonitis is an exceedingly rare entity in healthy children (1).

The mechanism of primary peritonitis remains unknown, although hematogenous, lymphatic, retrograde inoculation from the genitourinary tract and translocation of intestinal tract flora have all been proposed (1). In the adult population, retrograde inoculation via the genitourinary route has been speculated to cause GAS primary peritonitis. Several case reports describe adult females developing GAS primary peritonitis after oral sexual intercourse with partners who were shown to asymptomatically carry identical strains of GAS in the oropharynx $(2,3)$. In our case, the patient denied any type of sexual activity and, unfortunately, vaginal swabs were performed well into her antibiotic course; therefore, proof of an ascending route of infection was not achieved. However, because this patient did not have any other obvious source of infection, an ascending route of infection was still a possibility. Several cases of GAS primary peritonitis have also been associated with other household members having recent GAS pharyngitis. There was no history of this in our patient, and her pharyngeal swab, performed after antibiotics, yielded a negative result (4).

In healthy children, Streptococcus pneumoniae is the most common cause of primary peritonitis, although Staphylococcus aureus, GAS and Gram-negative organisms, such as Escherichia coli, have been observed (1). The most common predisposing factors are nephrotic syndrome, in which similar pathogens are seen, and cirrhosis complicated by portal hypertension, in which E coli, Klebsiella species and S pneumoniae are seen (1). In adolescent girls, gonococcal peritonitis must also be considered as a complication of pelvic inflammatory disease (1). Empirical coverage for primary peritonitis should include a third- or fourth-generation cephalosporin, a broad-spectrum penicillin or ampicillin plus an aminoglycoside (1).

Primary peritonitis is seen infrequently in the healthy pediatric population, and within that category, GAS primary peritonitis is an exceedingly rare disease. GAS is more typically associated with streptococcal pharyngitis, cellulitis and, less commonly, invasive disease such as necrotizing fasciitis. Review of the literature reveals only three reports of GAS primary peritonitis in the healthy pediatric population. Sewrey et al (5) describe a seven-month-old female infant with GAS primary peritonitis, with no obvious source, managed with laparotomy and antibiotic therapy. Dann et al (6) describe two previously healthy females, six and eight years of age, both of whom received similar operative and antibiotic management.

Primary GAS peritonitis occurs more commonly in adult females of reproductive age. A recent case series from the United Kingdom demonstrated that the six patients with the disease, the median age was 35 years, with five female cases and only one male case (7). These patients presented with a variety of symptoms, including fever, vomiting, diarrhea and influenza-like syndrome, and had peritoneal findings on examination $(4,7)$. Four of the cases were complicated by streptococcal TSS, as was seen in our case (7). GAS was most often isolated from blood and/or peritoneal fluid cultures, as was seen with our patient $(4,7)$.

Diagnostic imaging may prove useful in directing the management of GAS primary peritonitis. As described above, Dann et al (6) provided two case reports of GAS primary peritonitis in children. In the first case, a contrast-enhanced, abdominal-pelvic computed tomography (CT) scan demonstrated intraperitoneal fluid, moderately dilated loops of small bowel and peritoneal enhancement, with the appendix not visualized. These nonspecific findings were believed to be secondary to appendicitis, so a laparotomy was performed, from which peritoneal fluid was diagnostic for GAS primary peritonitis. Another case presented with similar CT findings, but this time with the appendix was visualized, showing only mild wall thickening. Because of the previous case, primary peritonitis was included on the differential, and the child was managed less invasively with laparoscopy, during which primary peritonitis was diagnosed. Dann et al (6) suggested that if imaging findings of peritonitis are much more significant than the degree of appendiceal abnormality, primary peritonitis should be included on the differential, in which case the diagnosis may be made via paracentesis instead of invasive surgery and managed medically with antibiotics. However, there are no clinical cases to support this approach.

In contrast to this recommendation, a recent literature review of 10 adult patients with GAS primary peritonitis described most patients receiving CT scans of the abdomen, with only one patient avoiding surgical exploration (4). Because these patients may present acutely ill with TSS, and secondary causes of peritonitis cannot be ruled out definitively with diagnostic imaging, conservative management with paracentesis and antibiotic therapy may not be a practical or safe option alone. In our patient, the appendix was not visualized despite the presence of free fluid, making surgical exploration a necessity.

Due to the limited number of cases, no standardized guidelines exist for management of GAS primary peritonitis. Because it is rarely suspected, empirical antibiotic therapy with broad-spectrum antibiotics covering intestinal flora is typically started initially, as was seen with our patient (4). As described above, imaging findings may be nonspecific, leading to exploratory laparotomy to rule out appendicitis. Once GAS is isolated from culture, penicillin or another beta-lactam antibiotic is typically initiated $(4,7)$. In the case of TSS, clindamycin, IVIG and intensive care may also be provided, as with our patient (4).

In summary, we describe a previously healthy 14-year-old girl, who presented with GAS primary peritonitis and TSS. GAS primary peritonitis should be considered in the differential diagnosis for acute abdominal pain in the pediatric population in cases where peritoneal findings on diagnostic imaging are much more significant and out of keeping with the appearance of the appendix as the source. In these cases, patients require prompt antibiotic therapy and, if toxic shock is present, intensive care treatment, clindamycin and potentially IVIG. Surgical therapy may be unavoidable if the patient has nonspecific findings on diagnostic imaging. The present report highlights the diagnostic and treatment dilemmas associated with primary peritonitis in the pediatric population.

ACKNOWLEDGEMENTS: No financial support was obtained for this article. RAH and AM drafted the manuscript. TK critically revised the manuscript for content. All authors saw and approved the final manuscript. All authors declare no conflicts of interest. TK is supported in part by a Career Award in the Biomedical Sciences from the Burroughs Wellcome Fund, and by a CIHR Training Grant in the Canadian Child Health Clinician Scientist Program, in partnership with Sick Kids Foundation, Child \& Family Research Institute (British Columbia), Women \& Children's Health Research Institute (Alberta) and the Manitoba Institute of Child Health.

\section{REFERENCES}

1. Rangel SJ, Moss RL. Chapter 69: Peritonitis. In: Long SS, Pickering LK, Prober CG, eds. Principles and Practice of Pediatric Infectious Diseases, 3rd edn. Churchill Livingstone - An Imprint of Elsevier Science, 2009:420.

2. Manalo R, Mirza H, Opal S. Streptococcus pyogenes tuboovarian abscess: A potential sexually transmitted disease? Sex Transm Dis 2002;29:606-7.

3. Gisser JM, Fields MC, Pick N, Moses AE, Srugo I. Invasive group A streptococcus associated with an intrauterine device and oral sex. Sex Transm Dis 2002;29:483-5.

4. Tilanus AM, de Geus HR, Rijnders BJ, Dwarkasing RS, van der Hoven B, Bakker J. Severe group A streptococcal toxic shock syndrome presenting as primary peritonitis: A case report and brief review of the literature. Int J Infect Dis 2010;14(Suppl 3):e208-12.

5. Sewrey H, Bryant PA. Group A streptococcus causing primary peritonitis in a healthy infant. Pediatr Infect Dis J 2009;28:1146.

6. Dann PH, Amodio JB, Rivera R, Fefferman NR. Primary bacterial peritonitis in otherwise healthy children: Imaging findings. Pediatr Radiol 2005;35:198-201.

7. Monneuse O, Tissot E, Gruner L, et al. Diagnosis and treatment of spontaneous group A streptococcal peritonitis. Br J Surg 2010;97:104-8. 


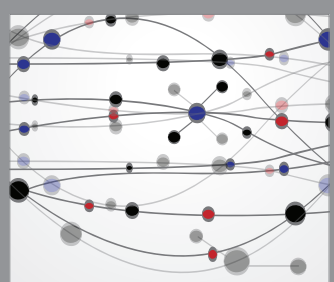

The Scientific World Journal
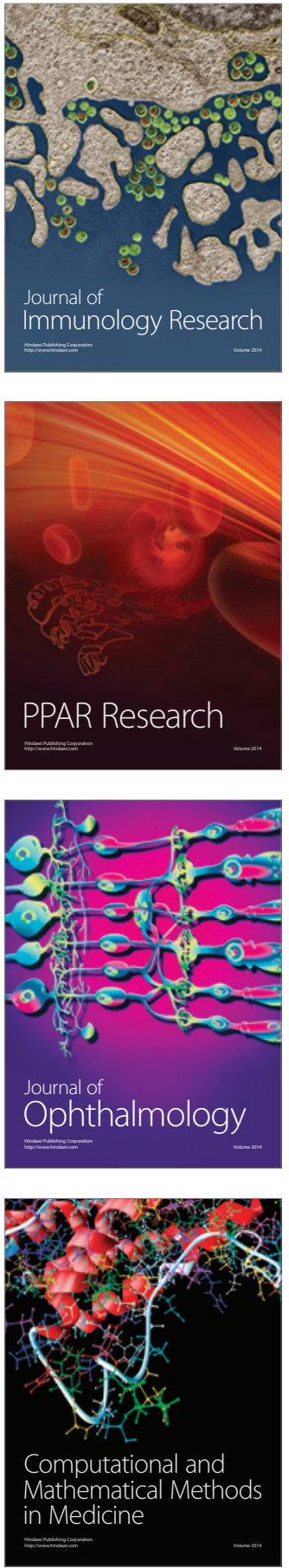

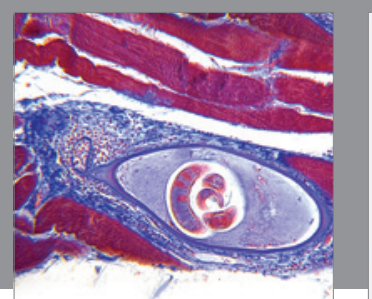

Gastroenterology Research and Practice

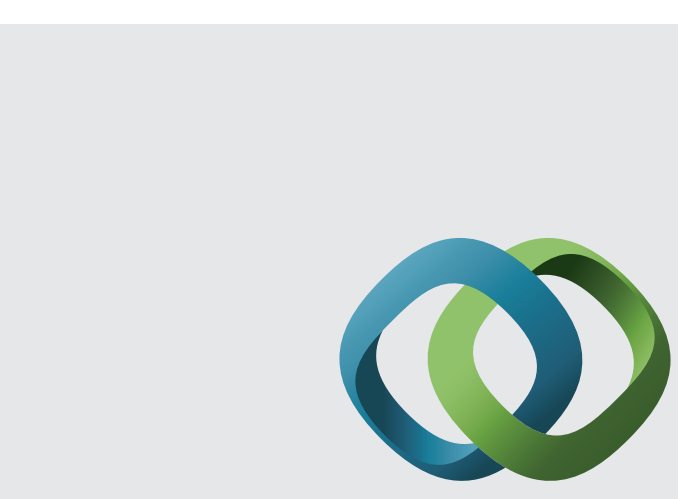

\section{Hindawi}

Submit your manuscripts at

http://www.hindawi.com
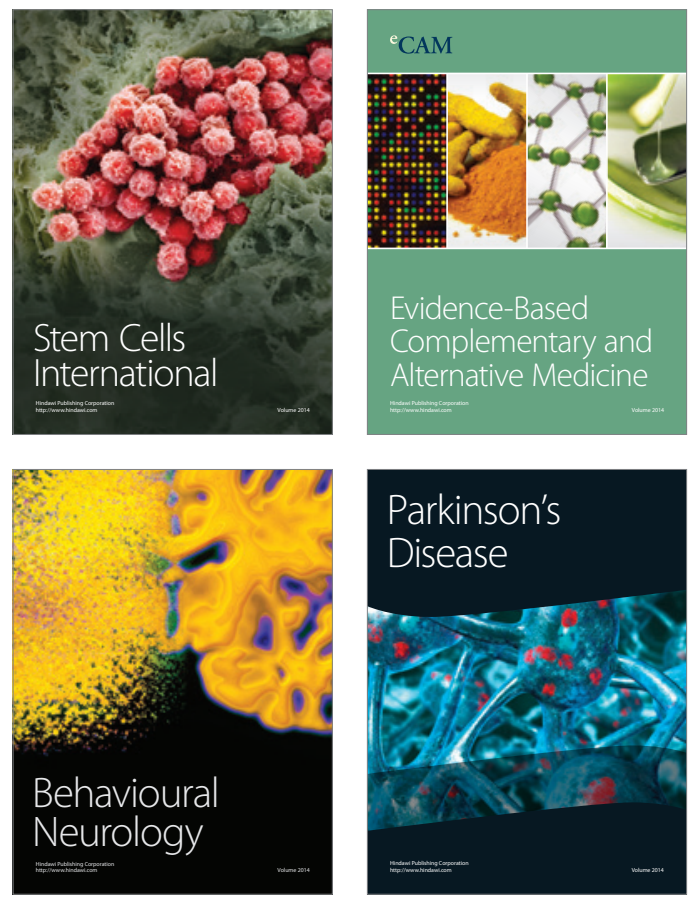
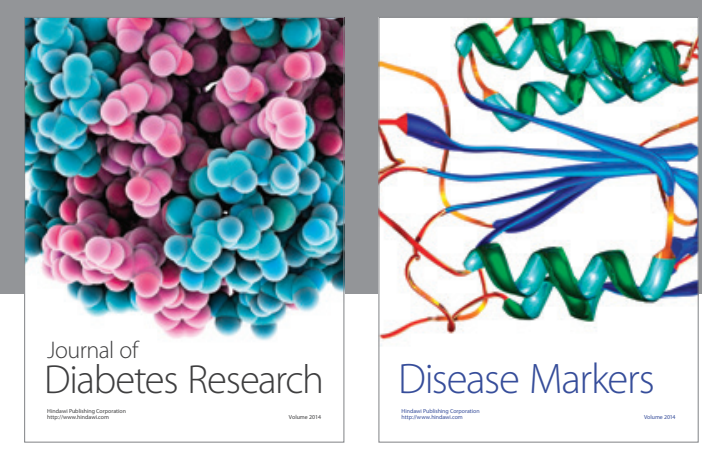

Disease Markers
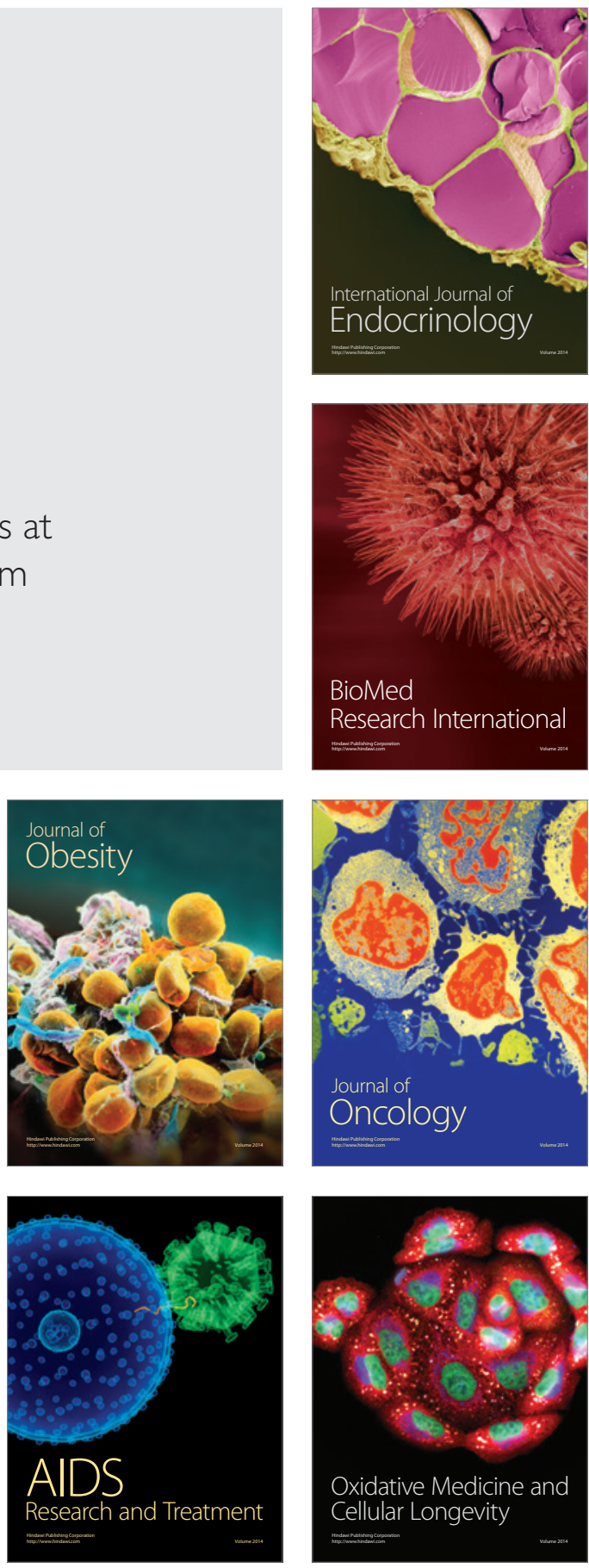